\title{
A Possible Interaction of Voriconazole and Warfarin in a Renal Transplant Recipient
}

\author{
A. Scott Mathis* and Daryl S. Schiller
}

Department of Pharmacy, Saint Barnabas Medical Center, Livingston, New Jersey, USA

\begin{abstract}
A potential warfarin - voriconazole interaction has been identified in one study involving healthy individuals. We report a case where addition of voriconazole possibly increased the international normalized ratio (INR) in a renal transplant patient taking warfarin. The INR elevation was resistant to reversal agents, and the situation may have ultimately contributed to a thrombotic episode.
\end{abstract}

\section{INTRODUCTION}

Voriconazole is a triazole antifungal derivative of fluconazole with enhanced potency against fungal cytochrome P(CYP)450-dependent 14 $\alpha$-sterol demethylase. Consequently, voriconazole has enhanced activity against Aspergillus fumigatus and Candida krusei. Based upon their mechanism of action, azole antifungals undergo extensive hepatic metabolism. Voriconazole is metabolized primarily by CYP2C19, but also CYP2C9 and CYP3A4. The $N$-oxide metabolite of voriconazole inhibits CYP2C9 and CYP3A4 to a greater extent than CYP2C19 [1,2]. These data suggest the potential for numerous drug-drug interactions with voriconazole, including an interaction with warfarin. One study conducted in healthy males evaluated the pharmacodynamic effects of the addition of a single dose of warfarin to twelve days of oral voriconazole in a placebo-controlled two-way crossover design and demonstrated a prolongation of prothrombin time/international normalized ratio (INR) when warfarin was administered with voriconazole [3]. To our knowledge there is no additional data evaluating the warfarin-voriconazole interaction. A significant drug-drug interaction could result in difficulty maintaining an INR within acceptable range, and potential downstream consequences, such as bleeding, thrombosis, delays in procedures, or prolonged hospitalization [4]. We report a case describing a hospitalized patient who may have experienced a clinically significant warfarin-voriconazole drug-drug interaction resulting in INR elevation resistant to reversal agents, cancellation of a procedure, and a thrombotic episode after the INR declined. Institutional Review Board approval was granted for reporting this study.

\section{CASE}

A 74 year old male with a cadaveric renal transplant 4 years prior was admitted with a four day history of fever, chills, headaches, generalized body aches, loss of appetite and mild nausea. There was no cough, sore throat, sinus

\footnotetext{
*Address correspondence to this author at the Department of Pharmacy, Saint Barnabas Medical Center, Livingston, New Jersey, USA;

E-mail: SMathis@sbhcs.com
}

pain, earache or urinary symptoms. The patient had a history of end stage renal disease due to hypertension, and prostate cancer, dyslipidemia, osteoporosis, chronic atrial fibrillation and a recent herpes zoster infection. For the transplant, the patient received basiliximab induction and maintenance immunosuppression with cyclosporine, sirolimus and prednisone. The patient took nifedipine and metoprolol for hypertension, doxazosin for the prostate and hypertension, simvastatin for dyslipidemia, alendronate for osteoporosis, famciclovir for herpes zoster, and amiodarone, warfarin and lowdose aspirin for the atrial fibrillation. Upon admission, the temperature was 102.4 degrees Fahrenheit, pulse was 69 beats per minute and consistent with atrial fibrillation (irregular), blood pressure was $128 / 72 \mathrm{mmHg}$, respirations were 20 per minute, and oxygen saturation was $98 \%$. Physical examination revealed a resolving shingles rash on the right flank. Laboratory analysis demonstrated a white blood cell count of 10,100 per $\mathrm{mm}^{3}$ with $82.1 \%$ neutrophils, $4.4 \%$ lymphocytes, and $13.3 \%$ monocytes, and a platelet count of 132,000 cells per $\mathrm{mm}^{3}$. Sodium was $129 \mathrm{mEq} / \mathrm{L}$, chloride was $93 \mathrm{mEq} / \mathrm{L}$, serum creatinine was $1.3 \mathrm{mg} / \mathrm{dL}, \mathrm{CO}_{2}$ was 22 $\mathrm{mEq} / \mathrm{L}$, and laboratory results were otherwise unremarkable. A chest radiograph demonstrated a right lower lobe infiltrate.

The patient was presumed to have community acquired pneumonia and was started on ceftriaxone and azithromycin (Table 1). Alendronate and famciclovir were not continued after admission. On the second day a plasma cytomegalovirus polymerase chain reaction test was sent, which was negative. On the third day of admission the patient had an episode of chest pain which resolved with sublingual nitroglycerin, but no electrocardiogram changes were observed, and creatine-kinase-MB (CK-MB) fraction and troponin $\mathrm{T}$ remained within normal limits. On the fourth day a chest radiograph demonstrated bilateral interstitial infiltrates with patchy alveolar infiltrates superimposed, most pronounced in the right lower lobe, and scattered nodular densities. On the sixth day chest radiograph revealed a bilateral parenchymal ground glass appearance in the upper and lower lobes, predominating in the upper lobes with extensive bullous changes suggestive of pneumonitis. On the seventh day cotrimoxazole was initiated for possible Pneumocystis carinii, 
Table. 1. Review of Medications Given (Black Bars) before, During (Grey Box), and after the Voriconazole-Warfarin Interaction

\begin{tabular}{|c|c|c|c|c|c|c|c|c|c|c|c|c|c|c|c|c|c|c|c|}
\hline & \multicolumn{19}{|c|}{ Day of Hospitalization } \\
\hline & 1 & 2 & 3 & 4 & 5 & 6 & 7 & 8 & 9 & 10 & 11 & 12 & 13 & 14 & 15 & 16 & 17 & 18 & 19 \\
\hline \multicolumn{20}{|l|}{ Immunosuppressives } \\
\hline \multicolumn{20}{|l|}{ Cyclosporine 50mg PO BID } \\
\hline \multicolumn{20}{|l|}{ Cyclosporine $25 \mathrm{mg}$ PO BID } \\
\hline \multicolumn{20}{|l|}{ Cyclosporine $75 \mathrm{mg}$ PO BID } \\
\hline \multicolumn{20}{|l|}{ Cyclosporine $100 \mathrm{mg}$ PO BID } \\
\hline \multicolumn{20}{|l|}{ Cyclosporine $60 \mathrm{mg}$ IV } \\
\hline \multicolumn{20}{|l|}{ Sirolimus 1mg daily } \\
\hline \multicolumn{20}{|l|}{ Prednisone $5 \mathrm{mg}$ daily } \\
\hline \multicolumn{20}{|c|}{ Methylprednisolone $20 \mathrm{mg}$ IV daily } \\
\hline \multicolumn{20}{|c|}{ Anticoagulants and Reversal Agents } \\
\hline \multicolumn{20}{|l|}{ Warfarin (See Fig. 1 for dose) } \\
\hline \multicolumn{20}{|l|}{ Enoxaparin 60mg SQ x1 } \\
\hline \multicolumn{20}{|l|}{ Enoxaparin 70mg SQ BID } \\
\hline \multicolumn{20}{|l|}{ Fresh Frozen Plasma } \\
\hline \multicolumn{20}{|l|}{ Vitamin K 5mg SQ } \\
\hline \multicolumn{20}{|l|}{ Cardiovascular } \\
\hline \multicolumn{20}{|l|}{ Metoprolol 100mg PO BID } \\
\hline \multicolumn{20}{|l|}{ Aspirin $81 \mathrm{mg}$ PO daily } \\
\hline \multicolumn{20}{|l|}{ Doxazosin PO 1mg AM, 2mg PM } \\
\hline \multicolumn{20}{|l|}{ Nifedipine XL 30mg PO Daily } \\
\hline Amiodarone $200 \mathrm{mg}$ PO dail & & & & & & & & & & & & & & & & & & & \\
\hline Simvastatin $20 \mathrm{mg}$ daily & & & & & & & & & & & & & & & & & & & \\
\hline Diltiazem 30mg q6h & & & & & & & & & & & & & & & & & & & \\
\hline Nitro tab $0.4 \mathrm{mg} \mathrm{x} 2$ & & & & & & & & & & & & & & & & & & & \\
\hline Furosemide $40 \mathrm{mg}$ IV or PO da & & & & & & & & & & & & & & & & & & & \\
\hline Infection & & & & & & & & & & & & & & & & & & & \\
\hline Ceftriaxone $1 \mathrm{~g}$ IV daily & & & & & & & & & & & & & & & & & & & \\
\hline Azithromycin $250 \mathrm{mg}$ PO dai & & & & & & & & & & & & & & & & & & & \\
\hline Azithromycin $500 \mathrm{mg}$ PO dai & & & & & & & & & & & & & & & & & & & \\
\hline Cotrimoxazole DS PO \#2 q12 & & & & & & & & & & & & & & & & & & & \\
\hline Levofloxacin 500mg IV dail & & & & & & & & & & & & & & & & & & & \\
\hline Vancomycin 1g IV daily & & & & & & & & & & & & & & & & & & & \\
\hline Meropenem 1g IV q8h & & & & & & & & & & & & & & & & & & & \\
\hline Doxycycline $100 \mathrm{mg}$ IV q12 & & & & & & & & & & & & & & & & & & & \\
\hline Acyclovir $600 \mathrm{mg}$ IV q8h & & & & & & & & & & & & & & & & & & & \\
\hline
\end{tabular}


(Table 1) contd...

\begin{tabular}{|c|c|c|c|c|c|c|c|c|c|c|c|c|c|c|c|c|c|c|c|}
\hline & 1 & 2 & 3 & 4 & 5 & 6 & 7 & 8 & 9 & 10 & 11 & 12 & 13 & 14 & 15 & 16 & 17 & 18 & 19 \\
\hline \multicolumn{20}{|c|}{ Voriconazole $300 \mathrm{mg}$ IV $\mathrm{x} 1$ in PM } \\
\hline \multicolumn{20}{|l|}{ Voriconazole $300 \mathrm{mg}$ IV q12h } \\
\hline \multicolumn{20}{|c|}{ Levalbuterol $0.63 \mathrm{mg}$ inhaled q6h } \\
\hline \multicolumn{20}{|l|}{ Ipratropium $0.5 \mathrm{mg}$ inhaled $\mathrm{q} 3 \mathrm{~h}$} \\
\hline \multicolumn{20}{|c|}{ Acetylcysteine $20 \% 3 \mathrm{~mL}$ inhaled $\mathrm{q} 6 \mathrm{~h}$} \\
\hline \multicolumn{20}{|l|}{ Gastrointestinal } \\
\hline \multicolumn{20}{|l|}{ Central Nervous System } \\
\hline \multicolumn{20}{|l|}{ Acetaminophen $650 \mathrm{mg}$ q6h prn } \\
\hline \multicolumn{20}{|c|}{ Propofol/vecuronium/hydromorphone IV } \\
\hline \multicolumn{20}{|l|}{ Other } \\
\hline Darbepoetin 200mcg SQ & & & & & & & & & & & & & & & & & & & \\
\hline
\end{tabular}

but a bronchial washing done on that day revealed benign bronchial and squamous epithelial cells in a background of macrophages and inflammatory cells with no viral inclusions or evidence of pneumocystis or fungal organisms.

Cotrimoxazole was subsequently discontinued. Separate staining for Mycoplasma pneumoniae was also negative. Tests for Legionella were negative. On the eighth day the patient was transferred to the intensive care unit due to worsening respiratory status. Sirolimus was discontinued on that day as a possible cause of pneumonitis. Ceftriaxone and azithromycin were switched to levofloxacin, meropenem and vancomycin. On the tenth day of hospitalization hemodynamic instability ensued and the antihypertensives were discontinued. Amiodarone was also stopped since it was considered a potential cause of the pneumonitis, and diltiazem was added for rate control. A chest radiograph demonstrated bilateral pulmonary opacities representing multilobar pneumonia, and the following day another radiograph demonstrated ill-defined parenchymal densities bilaterally, possibly representing tumor, pneumonitis, or pneumonia. On the twelfth day of hospitalization intravenous acyclovir and voriconazole were added since the patient's status was not improving and a sputum culture yielded a preliminary result of light candida species growth. The final culture results were presumptive Candida albicans and Enterococcus faecium susceptible to vancomycin. Another chest radiograph demonstrated increased left lower lobe infiltrates, and a computed tomography revealed extensive ground glass opacities throughout the lungs and bullous emphysematous lesions throughout the lungs, with right greater than left pleural effusion. The patient also underwent elective intubation on day 12. Subsequent chest radiographs during the ad- mission revealed no important changes. Blood cultures throughout the admission were negative. Serum creatinine remained stable between 1 and $1.5 \mathrm{mg} / \mathrm{dL}$ until day 26 .

The patient was on warfarin for prevention of thromboembolic complications associated with atrial fibrillation during the hospitalization. The INR was maintained at approximately 1.5-1.7 after an initial downward adjustment for an elevated INR upon admission (Fig. 1). The warfarin dose was increased after the amiodarone was discontinued (goal INR 2-3). However a lung biopsy was planned for inpatient day 14. After increasing the warfarin dose the INR was 3, at which time (day 12) voriconazole was started and two units of fresh frozen plasma (FFP) were given. Subsequently, the INR increased to 4.1 and then 5.8 over the next two days, despite holding warfarin and giving doses of subcutaneous vitamin $\mathrm{K}$ and an additional 2 units of FFP on day 14. After the INR reached 5.8 it declined. The patient's hemoglobin decreased $3 \mathrm{~g} / \mathrm{dL}$ during the period of the elevated INR, but no obvious source of bleeding was identified. The lung biopsy was delayed due to the elevated INR, and then ultimately cancelled due to worsening of the patient's clinical status. The drop in INR was observed 4 days after the last dose of warfarin. However, the INR only decreased to 1.9 over the next few days, despite stopping warfarin and administering FFP and vitamin K. On day 17 the patient developed a left upper extremity swelling and was diagnosed with an ultrasound-confirmed deep vein thrombosis, which was treated with enoxaparin. From admission until the peak INR, serum glutamic oxaloacetic transaminase (SGOT) ranged from 21 to 64 international units (IU) per liter and serum glutamic pyruvic transaminase (SGPT) ranged from 13 to 76 IU/L. The SGOT and SGPT during the period of the INR 


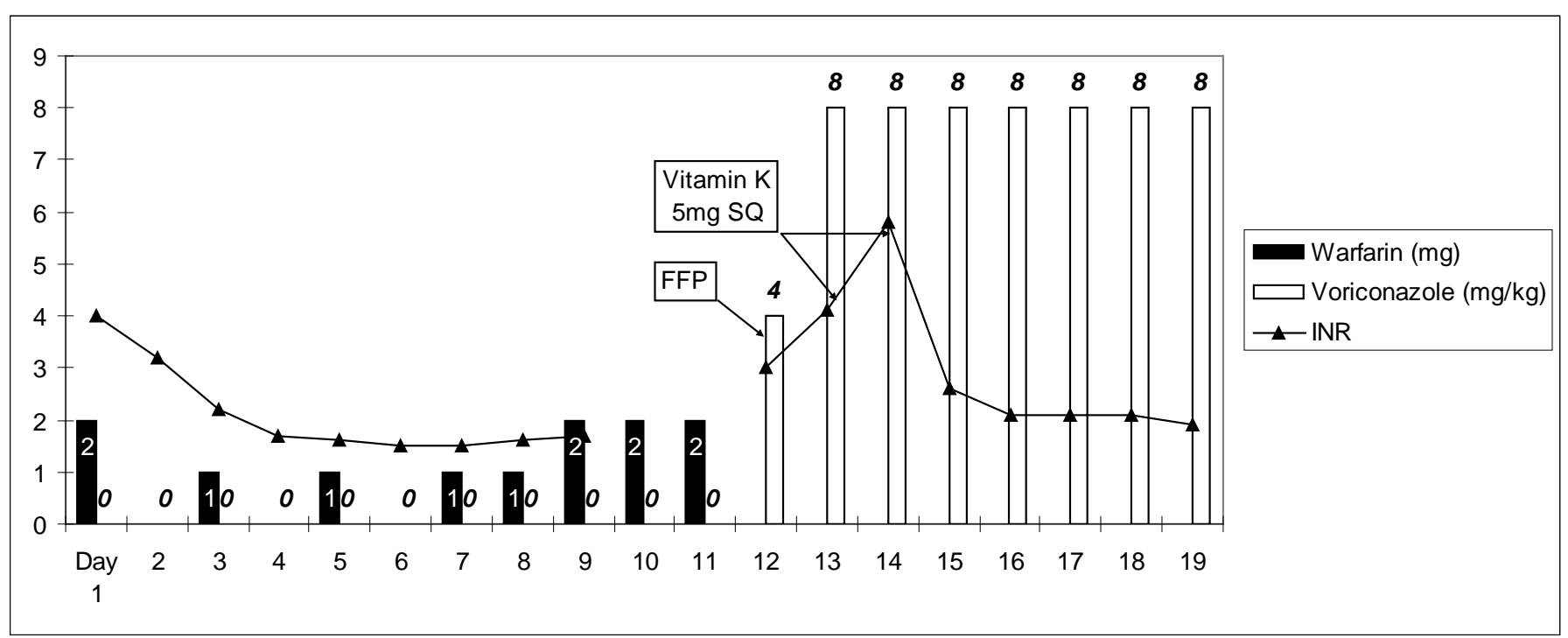

Fig. (1). Dosing of warfarin (black bars) in $\mathrm{mg}$ and voriconazole (white bars) in $\mathrm{mg} / \mathrm{kg}$ relative to INR (line with triangles). All use the same axis values on the left.

elevation were 24 to $40 \mathrm{IU} / \mathrm{L}$ and 26 to $61 \mathrm{IU} / \mathrm{L}$, respectively. For the next 10 days after the peak INR the SGOT was 21 to $76 \mathrm{IU} / \mathrm{L}$ and the SGPT was 23 to $62 \mathrm{IU} / \mathrm{L}$. On inpatient day 20 an abdominal film demonstrated considerable gallbladder sludge without stones or liver abnormality. However on day 25 , the amylase was 253 IU/L, lipase was 56 $\mathrm{U} / \mathrm{L}$, and the SGOT and SGPT increased to 1,110 and 936 IU/L, respectively. The patient remained unable to be weaned from the ventilator and became increasingly dependent on vasopressor agents for blood pressure support. A gastrointestinal bleed and a sepsis-like syndrome developed with continuing fevers and low blood pressure. On day 25 the CK-MB was elevated to $17 \mathrm{ng} / \mathrm{mL}$ and the troponin $\mathrm{T}$ was $0.235 \mathrm{ng} / \mathrm{mL}$, consistent with a non-ST-segment elevation myocardial infarction. The serum creatinine began to rise above $1.5 \mathrm{mg} / \mathrm{dL}$ on day 26 , going to $1.7 \mathrm{mg} / \mathrm{dL}$ and then to $2.4 \mathrm{mg} / \mathrm{dL}$ on the last day of hospitalization. The patient ultimately expired due to sepsis, respiratory failure, renal failure and hepatic failure.

\section{DISCUSSION}

Warfarin is an effective oral anticoagulant that suffers from numerous drug-drug and drug-food interactions that either potentiate the bleeding effects or reverse the anticoagulant effects [4,5]. Patients require many more medications than healthy individuals, so it is difficult to achieve target INR levels in patients with multiple comorbidities and medications. One study conducted in healthy individuals previously demonstrated that the hypoprothrombinemic effect of a single dose of warfarin was potentiated by daily voriconazole therapy [3]. We demonstrate for the first time, to our knowledge, an increase in the INR, decrease in hemoglobin and ultimately development of deep vein thrombosis when intravenous voriconazole was added after warfarin therapy in a patient taking multiple medications and with many medical conditions.

Warfarin is racemic mixture of R-warfarin and S-warfarin. R-warfarin is a weak inhibitor of vitamin $\mathrm{K}$ epoxide reductase and is metabolized to inactive metabolites primarily by CYP1A2 and CYP3A4. S-warfarin is the stronger inhibitor of vitamin K-dependent clotting factor synthesis and is mainly metabolized, under polymorphic genetic control, to inactive metabolites by CYP2C9 [6-9]. Voriconazole also has genotype-dependent dose requirements (CYP2C19) and has the ability to inhibit the metabolism of R-, and Swarfarin by inhibiting CYP3A4 and CYP2C9, respectively $[1,2]$. The pharmacokinetics of voriconazole are non-linear, so the half-life is dose-dependent and unpredictable, but in general about 6 hours. However, maximum concentrations are achieved within 1-2 hours [10].

The dose of warfarin was increased 3 days prior to initiation of voriconazole, and the last dose of warfarin was given approximately 24 hours before voriconazole initiation. The effects of warfarin dose modifications on INR are usually observed within 72 hours, which decreases the likelihood that the effect of adjusting the warfarin dose fully explains the rise in INR and resistance to reversal agents [4]. Unfortunately, INRs were unavailable on days 10 and 11 , the two days after the warfarin dose was increased, but before initiation of voriconazole.

Many other drug-drug interactions had the ability to potentiate or inhibit the antiprothrombinemic effects of warfarin in this case, so it is necessary to examine those possible relationships. Amiodarone, aspirin, azithromycin, doxycycline, cotrimoxazole, and simvastatin all had the potential to potentiate the warfarin effect, but all were stopped prior to voriconazole administration [4,5,11,12]. The elimination half-life of aspirin (3-10 hours) rules out its effect, but the elimination half-lives of simvastatin (unknown), doxycycline (15-20 hours), cotrimoxazole (15-20 hours), and azithromycin (70 hours) do not entirely rule out the possiblity of their contribution to an interaction [11]. However, the drugs were given concurrently with warfarin, so it is likely that their influence was accounted for by the warfarin dose.

Sirolimus, which has not been identified to interact with warfarin, and amiodarone, were both stopped due to their 
established relationship with pneumonitis $[13,14]$. Since a lung biopsy was cancelled as a result of difficulty managing the anticoagulation, the diagnosis was not established. The dose of warfarin had been increased prior to voriconazole administration, because of the amiodarone discontinuation and the subtherapeutic INR. Amiodarone, which has a highly probable association to clinically significant potentiation of warfarin was replaced with diltiazem. Diltiazem decreases $\mathrm{R}$-warfarin clearance, with clearance of S-warfarin unaffected, so it does not appear to prolong prothrombin time $[15,16]$. Amiodarone has a long half-life, so it is impossible to exclude an interaction with warfarin even after discontinuation. However, since the patient was on amiodarone longterm prior to admission we feel that full effects of that drugdrug interaction were realized prior to voriconazole administration.

Other drugs known to potentiate warfarin were given prior to and during voriconazole therapy including acetaminophen, pantoprazole, levofloxacin, methylprednisolone and vancomycin $[4,5,11,17,18]$. The relationship between levofloxacin and elevated INR in patients taking warfarin is controversial. Evidence suggests the possibility of a warfarin-levofloxacin interaction, but the data are inconsistent, and the effect only appears to occur in some patients. When a levofloxacin-warfarin interaction does occurs, the INR elevation appears to manifest at day 5-6 of combined therapy [18]. Use of pantoprazole, acetaminophen, methylprednisolone, and voncomycin were relatively consistent prior to and up until the use of voriconazole.

Furosemide and cyclosporine, drugs that could potentially decrease the effects of warfarin, were given prior to, and concurrently with voriconazole [4]. Propofol, a drug with a possible, but highly improbable, association to decrease the effects of warfarin was also given currently with voriconazole [4,5,11]. Vitamin K and FFP were given during voriconazole administration, and did not appear to take any effect until four days after warfarin was discontinued. The expected effect of holding warfarin dose and administration of FFP and vitamin $\mathrm{K}$ would be a reduction in INR within 24-48 hours [4]. Because of the long half-life of warfarin (36-42 hours) and its 2-5 day duration of action, the time frame of INR decline in this case was more consistent with diminishing effects of warfarin, rather than the effect of the antagonists [4]. However, despite the reduction of INR after 4 days, the INR remained $\sim 2$ for several more days without additional warfarin while voriconazole was continued. It is unlikely that a single dose of enoxaparin produced an elevation of INR during that time. Unfortunately, a deep vein thrombosis developed even with an INR of $\sim 2$. Liver disease also developed in this patient, but it was approximately 2 weeks after initiation of voriconazole, so the association with elevated INR was doubtful. A relationship between voriconazole and the liver disease was not established.

The drug-drug interactions, other than the voriconazolewarfarin interaction, do not appear to adequately explain the circumstances that occurred in this patient. Many of the drugs with well established relationships to warfarin potentiation were stopped prior to voriconazole administration. Other drugs that could interact with warfarin such as acetaminophen, pantoprazole, levofloxacin, methylprednisolone, vancomycin, furosemide and cyclosporine were given before and during voriconazole. However, since the potential levofloxacin-warfarin interaction manifests most commonly in the 5-6 day time-frame, the same time-frame from initiation of levofloxacin to initiation of voriconazole, the contribution of levofloxacin to the INR elevation cannot be ruled out entirely [18]. The only new drug with a potential interaction given during voriconazole therapy was propofol, a drug proposed to potentially decrease the anticoagulant effects of warfarin $[4,5]$.

\section{CONCLUSION}

This case has demonstrated for the first time a clinically significant potential drug-drug interaction between warfarin and intravenous voriconazole in a multi-morbid patient. The interaction produced an increase in INR even after discontinuation of warfarin, and administration of vitamin $\mathrm{K}$ and FFP, and resulted in cancellation of a procedure, a $3 \mathrm{~g} / \mathrm{dL}$ decrease in hemoglobin and ultimately contributed to the development of a deep vein thrombosis. Clinicians should be aware of the possibility of a clinically significant warfarinvoriconazole interaction, and enhance INR monitoring accordingly.

\section{REFERENCES}

[1] Pearson MM, Rogers PD, Cleary JD, Chapman SW. Voriconazole: a new triazole antifungal agent. Ann Pharmacother 2003; 37: 42032.

[2] Hyland R, Jones BC, Smith DA. Indentification of the cytochrome P450 enzymes involved in the $N$-oxidation of voriconazole. Drug Metab Dispos 2003; 31: 540-7.

[3] Purkins L, Wood N, Kleinermans D, Nichols D. Voriconazole potentiates warfarin-induced prothrombin time prologation. $\mathrm{Br} \mathrm{J}$ Clin Pharmacol 2003; 56: 24-9.

[4] Ansell J, Hirsh J, Hylek E, Jacobson A, Crowther M, Palareti G. Pharmacology and management of the vitamin $\mathrm{K}$ antagonists. The American college of chest physicians evidence-based clinical practice guidelines $8^{\text {th }}$ ed. Chest 2008; 133(Suppl): 160S-98S.

[5] Holbrook AM, Pereira JA, Labiris R, et al. Systematic overview of warfarin and its drug interactions. Arch Intern Med 2005; 165 : 1095-106.

[6] Lehmann DF. Enzymatic shunting: resolving the acetaminophenwarfarin controversy. Pharmacotherapy 2000; 20: 1464-8.

[7] Redman AR. Implications of cytochrome P450 2C9 polymorphism on warfarin metabolism and dosing. Pharmacotherapy 2001; 21: 235-42.

[8] Loebstein R, Yonath H, Peleg D, et al. Interindividual variability in sensitivity to warfarin- nature or nurture? Clin Pharmacol Ther 2001; 70: 159-64.

[9] Rieder MJ, Reiner AP, Gage BF, et al. Effect of VKORC1 haplotypes on transcriptional regulation and warfarin dose. $\mathrm{N}$ Engl $\mathrm{J}$ Med 2005; 352: 2285-93.

[10] Theuretzbacher U, Ihle F, Derendorf H. Pharmacokinetic/ pharmacodynamic profile of voriconazole. Clin Pharmacokinet 2006; 45 : 649-63.

[11] DrugDex Micromedex Healthcare Series. Thomson Scientific and Healthcare. Greenwood Village, CO 2006.

[12] Baciewicz AM, Bal BS. Bleeding associated with doxycycline and warfarin treatment. Arch Intern Med 2001; 161: 1231.

[13] Dusman EW, Stanton MS, Miles WM, et al. Clinical features of amiodarone-induced pulmonary toxicity. Circulation 1990; 82: 519.

[14] Champion L, Stern M, Israel-Biet D, et al. Brief communication: sirolimus-associated pneumonitis: 24 cases in renal transplant recipients. Ann Intern Med 2006; 144: 505-9.

[15] Abernethy DR, Kaminsky LS, Dickinson TH. Selective inhibition of warfarin metabolism by diltiazem in humans. J Pharmacol Exp Ther 1991; 257: 411-5.

[16] Stoysich AM, Lucas BD, Mohiuddin SM, Hilleman DE. Further elucidation of pharmacokinetic interaction between diltiazem and warfarin. Int J Clin Pharmacol Ther 1996; 34: 56-60. 
[17] Angaran DM, Dias VC, Arom KV, et al. The comparative influence of prophylactic antibiotics on the prothrombin response to warfarin in the postoperative prosthetic cardiac valve patient. Cefamandole, cefazolin, vancomycin. Ann Surg 1987; 206: 155-61.
[18] Carroll DN, Carroll DG. Interactions between warfarin and three commonly prescribed fluoroquinolones. Ann Pharmacother 2008; 42: 680-5.

(C) Mathis and Schiller; Licensee Bentham Open.

This is an open access article licensed under the terms of the Creative Commons Attribution Non-Commercial License (http://creativecommons.org/licenses/ by-nc/3.0/) which permits unrestricted, non-commercial use, distribution and reproduction in any medium, provided the work is properly cited. 